correspond directly to the permeability of the olivine matrix to metallic-melt percolation, the separation of a core from silicate mantle could happen very quickly in a planetesimal. The high temperatures required could result from the heat produced by the decay of shortlived isotopes present at the birth of the Solar System. More complete calculations of the thermal evolution of growing planetesimals (which include, for example, latent heat of melting, release of gravitational potential energy and impact kinetic energy) point to many sources of heat in the early Solar System that probably led to core formation and magma oceans in many growing planetesimals ${ }^{5}$.

But heating in a static environment may not be the whole answer. Deforming systems can have higher permeabilities than static systems, and impact-induced melting or differential stress may connect isolated melt pockets and produce pools of metal ${ }^{6}$ that may then sink through unmelted material. Each of these processes will tend to shorten the interval between accretion and core formation, so core formation should be ubiquitous once an accreting rocky planetesimal reaches a radius of $50-100 \mathrm{~km}$. But then the existence of large bodies that do not seem to have differentiated (including some large asteroids such as Ceres, and Jupiter's moon
Callisto) is puzzling: is there some mechanism that prevented these bodies heating sufficiently to produce a core?

Building on the success of Yoshino et al. ${ }^{1}$, future experiments may be able to determine melt connectivity through conductivity measured in situ and monitor dynamically evolving microstructure, such as during deformation or reactions. Synchrotron Xray microtomography ${ }^{7}$ is another promising technique, which enables three-dimensional imaging with resolution approaching $1 \mu \mathrm{m}^{3}$. These experimental advances will help us to understand the processes that have shaped the Solar System.

Bill Minarik is in the Department of Geology,

University of Maryland, College Park,

Maryland 20742, and at the Carnegie Institution

of Washington, Washington, DC 20015, USA.

e-mail:minarik@geol.umd.edu

1. Yoshino, T., Walter, M. J. \& Katsura, T. Nature 422, 154-157 (2003).

2. Yin, Q. et al. Nature 418, 949-952 (2002).

3. Kleine T., Münker, C., Mezger, K. \& Palme, H. Nature 418, 952-955 (2002).

4. von Bargen, N. \& Waff, H. S. J. Geophys. Res. 91, 9261-9276 (1986).

5. Ghosh, A. \& McSween, H. Y. Icarus 134, 187-206 (1998)

6. Bruhn, D., Growbner, N. \& Kohlstedt, D. L. Nature 403, 883-886 (2000).

7. Roberts, J. J., Tyburczy, J. A., Locke, D., Minarik, W. \& Kinney, J. Eos 83, F400 (2002).

Evolutionary biology

\title{
Teeth as tools
}

Anne Weil

What determines the shapes of mammalian teeth? When tools are designed to cut to the meat of the question, form follows function rather than developmental or evolutionary constraints.

V ery different groups of mammals have teeth of similar shapes. One obvious explanation for this is that the greatest efficiency in chewing similar foods is strongly favoured by natural selection. But other reasons could include the constraints imposed in the process of development, or the historical limitations imposed within mammal lineages, and each of these factors might act against or in concert with the demands of optimizing function.

Writing in the Biological Journal of the Linnean Society (78, 173-191; 2003), Alistair R. Evans and Gordon D. Sanson describe how they have taken an engineer's approach to this question. They have carried out a computer-modelling exercise, designing tools to cut tough substances, and find that the most efficient tools closely resemble the molars of carnivorous and insectivorous mammals. They conclude that in many cases developmental and evolutionary factors have not strongly influenced molar shape, and that function is indeed the primary determinant.

Most mammals use differentiated cheek

teeth for chewing, to divide food into small pieces that can be swallowed easily and digested efficiently. Mammalian teeth are replaced at most once in an individual's lifetime, so exact positioning of them is possible, allowing the cutting edges and points of upper and lower teeth to meet in a precise way. The hands, tongue and facial muscles are variously used to position food between the teeth. Tough (as opposed to brittle or soft) foods are divided by an initial puncture (or punctures), which is then extended into a longer cut. Chewing tough foods can thus be envisaged as a mechanical task in which the teeth act as simple, edged tools.

Evans and Sanson considered six functional factors used by engineers in tool design: sharpness of points; sharpness of blades; the angle between the blade and the substance cut; the angle between the blade and a line perpendicular to the cut; the entrapment of substance between blades; and the movement of substance away from the blade that prevents the implement from becoming clogged up. They considered these

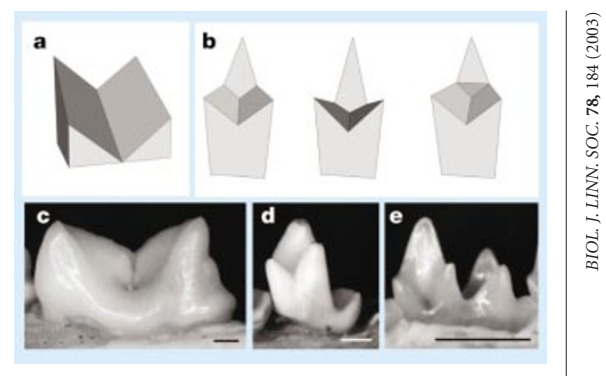

Figure 1 Efficient model cutting tools, and some similarly shaped mammalian cheek teeth. The single-bladed (a) and double-bladed (b) models optimize eight efficiency factors considered by Evans and Sanson. On that evidence, real mammalian molars (c, single-bladed; d, e, double-bladed) may approach a functional ideal. Scale bars, $1 \mathrm{~mm}$.

factors for tools with single blades (which are rectangular in horizontal cross-section, and can resemble a chisel, scissors or a guillotine), as well as for tools with two blades that meet at an angle (which are triangular in cross-section). Not surprisingly, they found that some shapes work better than others. The field of optimal shapes narrowed further when trueto-life criteria were applied: a serial arrangement for the blades, like that of teeth in the jaw, and a degree of lateral as well as vertical movement, as commonly occurs in chewing.

In the case of the single blades, the most efficient is a symmetrical, notched blade (Fig. 1a), strikingly similar to 'carnassial' teeth that have evolved in several mammal lineages (Fig. 1c). The optimal doublebladed models have three points and two high crests (Fig. 1b), forming a 'protoconoid' that closely resembles the trigonid of simple mammalian lower molars (Fig. 1d, e). This notched triangle is a familiar shape to any student of mammalian evolution, because it evolved early and possibly more than once in mammalian history and is present in many living groups, such as opossums and bats.

Evans and Sanson's study did not address the significant role of crushing in chewing. Their modelling therefore did not produce a 'tribosphenic' tooth shape, characterized on the lower molars by a low basin behind the high trigonid (Fig. 1d, e) into which the largest cusp of the upper tooth fits. Tribosphenic molars perform both slicing and crushing functions, and were present in the ancestors of all living mammals. Although Evans and Sanson focused on cutting alone, the superior efficiency of their protoconoid models, and the evident supremacy of function in determining tooth form, may support the arguments of those who believe that tribospheny evolved two or even three times within early mammals.

Anne Weil is in the Department of Biological Anthropology and Anatomy, Duke University, Durham, North Carolina 27708-0383, USA. e-mail:annew@duke.edu 